\title{
Tumor marker CA 15-3 in breast cancer patients
}

\author{
Hanifa Fejzić*, Svjetlana Mujagić, Sanida Azabagić, Mensura Burina
}

Department of Radiology and Nuclear Medicine, University Clinical Center Tuzla, Bosnia and Herzegovina

\footnotetext{
${ }^{*}$ Corresponding author: fejzic.hanifa@gmail.com Tel.: + 38735255405

Fax.: + 38735251456
}

Received: 12 March 2015

Accepted: 8 May 2015

Key words: Tumor marker CA 15-3 Breast cancer $\cdot$ Metastatic disease $\cdot \mathrm{BI}$ RADS classification

\begin{abstract}
Objective. The aim of this study was to determine whether there is a correlation between the serum concentration of the tumor marker CA 15-3 and breast cancer, which has not been proven by the existence of regional and distant metastases, and breast cancer with the presence of regional and distant metastases. Patients and methods. The study was a retrospective-prospective study, and was conducted on 100 women aged 40-70 years of age in the period of January 2007 until June 2011, in whom, after surgery, breast cancer was histologically verified, where before the surgery serum tumor marker CA 15-3 levels were established. The serum tumor marker CA 15-3 concentrations are determined in all patients after radiological diagnosis of suspected breast cancer (radiological findings concluded as BI RADS 4 and 5). The study excluded patients with liver cirrhosis, liver cancer and lung cancer. The study group consisted of patients with metastatic breast cancer, and the control group of patients with breast cancer comprised those shown to be without verified metastatic disease. To calculate the correlation, Spearman's correlation coefficient was used. A difference in $p$ values of less than $0.05(\mathrm{p}<0.05)$ was considered statistically significant. Results. The serum tumor marker CA 15-3 was elevated in all patients with proven remote or clubbing metastasis in $35.5 \%$ of patients with metastasis spreading to regional lymph nodes. Conclusion. There is a significant correlation between serum concentrations of the tumor marker CA 15-3 and the presence of metastasis, and serum concentrations of tumor markers and the dissemination of the underlying disease.
\end{abstract}

\section{Introduction}

Breast cancer is stage-biological process causing multiple genetic and epigenetic changes in the epithelial cells of the breast over a period of several years (1). The biological progression of breast cancer is based on the inability of differentiation, loss of contact inhibition, uncontrolled growth, the ability to migrate, invasion, angiogenesis, metastasis, and the ability to avoid immune control of tumor cells. Breast cancer tumor cells generally retain the morphologi- cal and functional characteristics of normal tissue cells. They possess special molecules that have arisen in the course of malignant transformation. Many of these new tumor cell molecules are immunogenic, i.e. they are able to induce a humoral and / or cellular immune response. These reactions occur in patients with cancer, or in animals immunized with breast cancer cells. These specific antigens are classified into two groups: tumorospecific (TSA) and tumoroassociated (associated) antigens (TAA) (1). 
Circulating tumor antigens and markers are shown in the serum. For this purpose only the monoclonal antibodies-TAA: CEA and CA 15-3 are used. CEA (carcinoembryonic antigen) is a heterogeneous glycoprotein, with a molecular weight of 150 to 300 $\mathrm{kDa}$, whose serum concentrations are abnormally elevated in patients with colorectal cancer, breast cancer and other cancers $(2,3)$. The values may be increased in heavy smokers and nonmalignant diseases (4). CA 15-3 is a glycoprotein with a molecular weight of $300-450 \mathrm{kDa}$. It is elevated in breast, ovarian, pancreatic, lung and colorectal cancers (5).

The European Group on Tumor Markers (EGTM) recommended determining serum levels of tumor markers during the follow-up of treated breast cancer patients (6). EGTM defines a significant increase in concentration of tumor markers if its value is increased at least $25 \%$ compared to the reference value.

The frequency and level of each tumor marker in the serum depends on the clinical extent of the disease. The frequency of positive findings was significantly higher in patients with metastatic compared to clinically confined disease. A comparative study of CEA and CA 15-3 shows that CA 15-3 is a more sensitive marker than CEA. The frequency of both markers was greatest in patients with metastatic lesions in the liver. Increased levels of the markers CEA and CA 15-3 can be detected on average 3-8 months before a definitive clinical diagnosis of metastases (1). Tests for determination of tumor antigens in serum have been proven to be a useful tool for monitoring treatment response in breast cancer patients (5).

The aim of this study was to determine whether there is a correlation between the serum concentration of the tumor marker CA 15-3 and primary breast cancer, where the existence of regional and distant metastases has not been shown, and breast cancer with the presence of regional and distant metastases, and thereby indicate the diagnostic value (or lack of value) of the tumor marker CA 15-3 in the detection of primary breast cancer.

\section{Patients and methods}

The study was a retrospective-prospective study, and was conducted on 100 women aged 40-70 years of age in the period from January 2007 to June 2011, in whom after surgery breast cancer was histologically verified, and for whom before the surgery serum tumor marker CA 15-3 concentrations were established. The study excluded patients with liver cirrhosis, liver cancer and lung cancer. Studies for serum tumor markers CA 15-3 were performed in those patients in whom ultrasound and mammography showed suspected breast cancer (radiological findings BI RADS 4 and 5). Patients were divided into two groups in relation to the presence of metastases: the study and the control group. The study group consisted of 50 patients in whom breast cancer had been histologically verified and in whom after surgical removal of the content of the axillary lymph nodes the existence was shown histologically of metastases in the regional lymph nodes, and other available diagnostic methods had proved the presence of distant metastases. Given the localization of secondary deposits, patients from the study group were divided into two subgroups: firstly the subgroup of patients in whom breast cancer was proven with metastases to regional lymph nodes by histopathological analysis of tissue samples, and secondly the subgroup patients in whom, alongside the presence of cancer in the breast and metastases to the regional lymph nodes, the presence of distant and associated metastases had been shown.

The control group consisted of 50 patients in whom breast cancer had been histologically verified, and in whom, after surgical removal of the contents of the axillary 
lymph nodes, the existence of metastases in the regional lymph nodes had been excluded by histopathology, and other available diagnostic methods had excluded the existence of distant metastases. Mammography and breast ultrasound examinations were performed at the Department of Radiology and Nuclear Medicine, Clinical Center, Tuzla. Mammography was performed using a digital mammography machine, Simens brand-Mammomat 3000 NOVA with cassette sizes $18 \times 24$ and $24 \times 30 \mathrm{~cm}$. Standard mammographic projections were made: craniocaudal and mediolateral.

Ultrasound examinations of the breasts were performed using the ultrasound machine "sonoline G60S" Siemens, with linear probes measuring 7.5 and $12 \mathrm{MHz}$. Mammography and breast ultrasound findings were interpreted by two radiologists and classified by BI RADS classification into one of five categories: 1 . Breasts where no pathological lesions are seen; 2 . Benign findings; 3. Probably benign findings; 4 . Lesions suspicious for malignancy; 5. Lesions highly suspicious for malignancy-malignant lesion.

In patients with changes in the breasts, whose ultrasound and mammography findings were classified as BI RADS 4 or $\mathrm{BI}$ RADS 5, serum tumor marker CA 15-3 concentrations were taken by sequential Chemiluminiscence Immulite 2000 BR-MA system analysis and quantitative measurement of serum levels of CA 15-3 antigens in human serum on $\mathrm{ABBOT}$ apparatus. The reference values of serum concentrations of tumor markers CA $15-3$ were 0 to $31.3 \mathrm{U} / \mathrm{ml}$. Significantly elevated values were considered to be values $25 \%$ greater than the upper reference value, or greater than $39.25 \mathrm{U} / \mathrm{ml}$.

\section{Statistical analysis}

For statistical analysis, we applied standard methods of descriptive statistics, such as: relative numbers (\%), measures of central tendency, measures of variability, parametric $\mathrm{z}$ and $\mathrm{t}$-test, and test of proportions. To test the statistical significance of differences between respondents we used the Student $t$ test. To calculate the correlation we used Spearman's correlation coefficient. The difference between the samples was considered significant when $\mathrm{p}$ was less than $0.05(\mathrm{p}<0.05)$.

\section{Results}

The study included 100 patients with histologically verified breast cancer, with an average age of $55.27 \pm 8.16$ years. The average age of the patients in the study group was $54.74 \pm 8.41$ years, while the control group was $55.8 \pm 7.94$ years old. There was no significant difference in age between the patients in the study and the control group ( $p>0.05$ ). Preoperative ultrasound and mammography findings in $28 \%$ patients were concluded as BI RADS 4 , and in $72 \%$ patients as $\mathrm{BI}$ RADS 5. In the control group of patients there were 50 patients with breast cancer detected by histopathologic analysis of tissue samples, and histopathological evaluation of the content of the axillary metastatic axillary lymph nodes had excluded the existence of metastases. In this group of patients the serum tumor marker CA 15-3 was within the reference values from 2.7 to $26.7 \mathrm{U} / \mathrm{ml}$.

All patients from the study group, 50 of them $(100 \%)$, had proven metastases in the regional lymph nodes, and 19 of them (38\%), alongside the proven metastases in the regional lymph nodes, also had distant metastases. In this group of patients significantly elevated levels of serum tumor marker CA 15-3 were also shown, with a mean value of 214.70 $\mathrm{U} / \mathrm{ml}$. Taking into account the localization of the metastatic processes, the statistically highest serum concentrations of the tumor marker CA 15-3 were detected in patients with metastasis in the bones, as well as in patients in whom metastatic processes were demonstrated in several organs or organ systems. 
In the group of patients with metastasis only in the regional lymph nodes, serum tumor marker CA 15-3 levels ranged from 11.2 $\mathrm{U} / \mathrm{ml}$ and $61.3 \mathrm{U} / \mathrm{ml}$. There was no statistically significant correlation between serum levels of tumor markers CA 15-3 and the number of axillary lymph nodes affected.

Taking into account the concentrations of the serum tumor marker CA 15-3 in the study group in $40 \%$ of them were within the reference values, in $10 \%$ of them were elevated, and in $50 \%$ of them they were significantly elevated. In the control group of patients, values of serum tumor marker CA 15-3 were within the reference values.

Student's t-test showed that concentrations of the serum tumor marker CA 15-3 were statistically significantly higher in the study group than in the control group $(p<0.0001)$. The same test showed that the concentrations of the serum tumor marker CA 15-3 were significantly lower in the study group than the threshold, indicating increased concentrations $(t=21.0$; $\mathrm{p}<0.0001$ ), whereas serum tumor marker CA 15-3 concentrations in the study group were significantly higher than the value that indicates significantly elevated concentrations of tumor markers CA 15-3 ( $\mathrm{t}=3.2$; $\mathrm{p}=0.001$ ). Student's $\mathrm{t}$-test showed that serum tumor marker CA 15-3 concentrations in the study group were significantly higher in the subgroup of patients with the presence of distant metastasis $(\mathrm{p}<0.0001)$ than in the subgroup of patients with metastases in the regional lymph nodes only.

The most common location of metastasis were the bones (52.6\%), followed in frequency by associated metastases, where secondary deposits could be found in two or more organs or organ systems $(31.6 \%)$. The incidence of associated metastases did not differ significantly statistically, as evidenced by the test of proportions $(\mathrm{z}=1.31 ; \mathrm{p}=0.189)$. Bone metastases were significantly more likely than those in other organs: the liver, CNS and lungs $(\mathrm{Z}=3.2 ; \mathrm{p}=0.0013)$. The same conclusion was reached by comparing the incidence of associated metastases and metastases in the CNS, liver and lungs $(\mathrm{Z}=2.9$; $\mathrm{p}=0.036)$ (Figure 1).

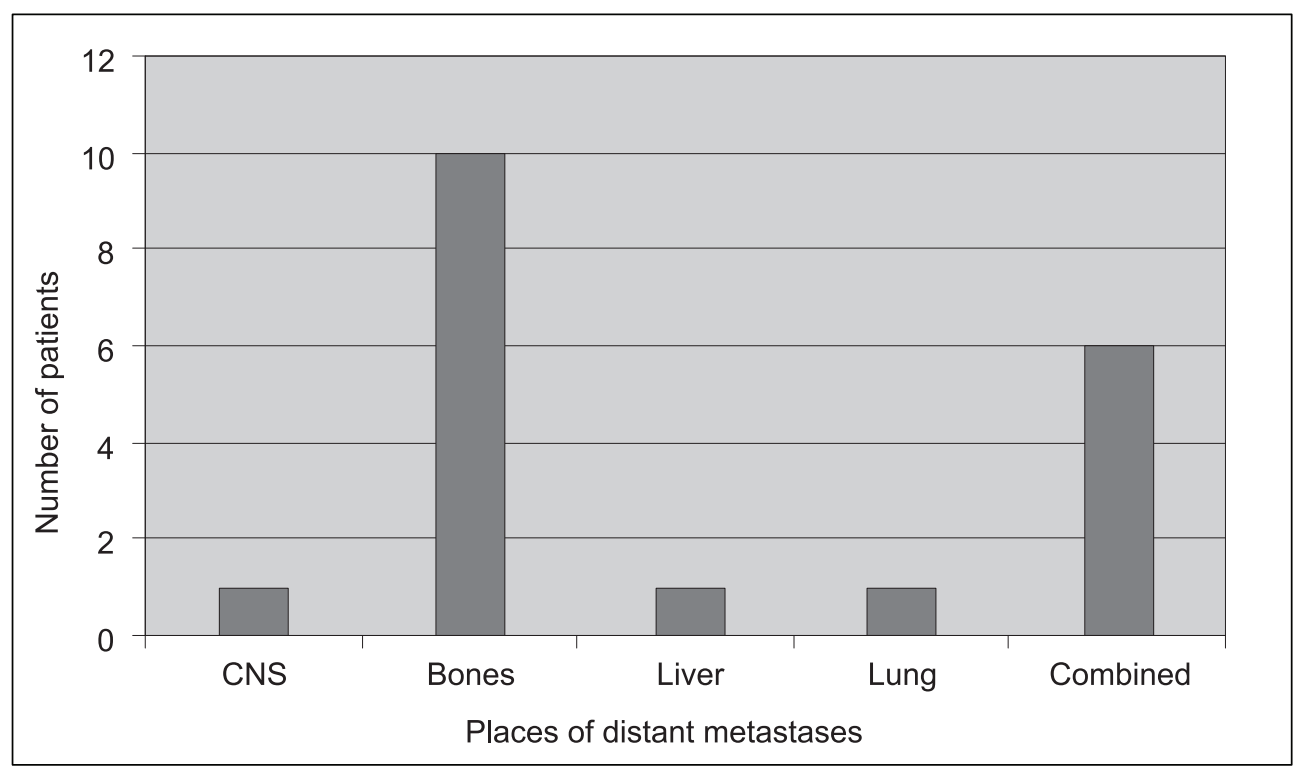

Figure 1. Distribution of patients with respect to the localization of distant metastases. 
Student's t-test showed that the concentrations of serum tumor marker CA 15-3 were statistically significantly higher in patients with associated distant metastases than in patients in whom metastases were present in the axillary lymph nodes only $(\mathrm{t}=4.5 ; \mathrm{p}=0.001)$.

A statistically significant positive correlation was found between serum levels of tumor markers CA 15-3 and the presence of distant metastases $(\rho=0.83 ; \mathrm{p}<0.0001)$.

\section{Discussion}

Numerous studies have confirmed that CA 15-3 and BR 27.29 are the best serum tumor markers of breast cancer available. The use of the currently available serum tumor markers is limited by their low sensitivity and specificity for early diagnosis. Consequently, the available markers are of no value in either screening or diagnosing early breast cancer (7).

However, serial measurement of these markers can result in the early detection of the recurrent disease, as well as indicating the efficacy of therapy (8). Other markers, mainly epitopes present on mucin glycoproteins (CA549, CA M26, CA M29, CA27.29) are under evaluation, but to date none have been shown to be more useful than CA 15-3. These other markers may be used instead of CA 15-3, but not in combination (9).

The sensitivity of tumor markers in the diagnosis of local recurrence is poor, but their usefulness (particularly that of CA 15-3) in the early diagnosis of breast cancer metastases is clear. The early detection of metastatic disease does not benefit the patient in terms of overall survival or time to the appearance of clinical signs (9).

Therefore, tumor markers are not recommended for any group of patients for screening, diagnosis, or monitoring of patients with breast cancer. Although high serum tumor marker CA 15-3 levels usually indi- cate an extended illness, not enough data are available to allow inclusion of tumor markers in routine monitoring of breast cancer. Both tumor marker CA 15-3 and BR 27.29 have "Food and Drug Administration" approval for monitoring patients suffering from breast cancer with advanced disease, but such authorization does not necessarily mean clinical value. The main obstacles in relation to the use of tumor markers CA 15-3 as indicators of asymptomatic recurrences include the low incidence of elevated serum concentrations of tumor marker CA $15-3$ in the early stages of the disease. Although the use of tumor markers CA 15-3 and BR 27.29 is not recommended in routine work and monitoring of breast cancer, but only for monitoring response to treatment, the guidelines of the American Society of Clinical Oncology (ASCO) support the use of these markers, since these tumor markers can indicate the progression of the disease, while all other measurable parameters are important in monitoring breast cancer using reference value ranges (10).

The National Academy of Clinical Biochemistry (NACB), EGTM, and SOR (the standards, options and recommendations offshore project in 1993, France) recommend that serum tumor markers CA 15-3 and BR 27.29 should be used with care, as an aid in monitoring the clinical disease in patients suffering from breast cancer. EGTM and SOR guidelines also recommend the use of serum concentrations of tumor markers CEA in patients with breast cancer.

The study by Vrdoljak et al. (11) which included 177 patients, investigated the relationship between serum levels of tumor marker CA 15-3 and metastases to regional lymph nodes in patients with breast cancer. In this study, $73 \%$ of patients with histologically verified metastases in regional lymph nodes had normal serum tumor markers CA 15-3, while $27 \%$ had elevated serum concentrations of tumor marker CA 15-3. In 
most studies conducted worldwide it is indicated that the value of tumor marker CA $15-3$ is elevated in the group of patients with advanced disease. The study by Berberoglu et al. (12), which was conducted on a cohort of 60 patients, in $68 \%$ of patients with positive lymph node metastasis in the axillary lymph nodes, elevated serum tumor marker CA 15-3 was present, while in the group of patients with distant metastases increased levels of serum tumor marker CA 15-3 were present in all patients (100\%).

According to the data, Berberogluoa et al. (12) serum tumor marker CA 15-3 had a mean value of 39.1 in the group of patients with proven metastases in the axillary lymph nodes, with deviation of $1.2 \mathrm{U} / \mathrm{ml}$. A study by the same authors concluded that the tumor marker CA $15-3$ is the tumor-specific marker in the diagnosis and especially the monitoring of breast cancer patients. The same authors suggest that elevated levels of the tumor marker CA 15-3 can be detected in $1 \%$ of the healthy population, $3 \%-20 \%$ of benign changes in the breast, $36 \%-40 \%$ of hepatitis and $14 \%-70 \%$ of cancer of the gastrointestinal tract and lungs.

The study by Laesing et al. (13), conducted in 2007 on a group of 119 patients with proven breast cancer and regional and distant metastases present, made a correlation between serum concentrations of tumor markers CA 15-3 and the localization of the metastases. Eighty patients (67.2\%) had metastases present only in one place, in the liver (19.3\%) or the bones (21.9\%). 39 patients had metastasis in multiple organs or organ systems. Patients with metastasis to the bones and liver had elevated serum tumor marker CA 15-3: with a median of $110 \mathrm{U} / \mathrm{ml}$ and $81 \mathrm{U} / \mathrm{ml}$, compared to patients with metastasis to the lungs and lymph nodes: median $48.4 \mathrm{U} / \mathrm{ml}$ and 20.2 $\mathrm{U} / \mathrm{ml}$. As for the correlation between the serum concentrations of tumor marker CA 15-3 and localization of metastases, the re- sults are often conflicting: research by Berruti et al. (14) showed that the prevalence of elevated serum levels of tumor markers CA 15-3 varies depending on the localization of the metastases. Patients with involvement of visceral organs had elevated serum tumor markers CA 15-3 more often than patients with involvement of bones and soft tissue. The highest sensitivity, in terms of elevated serum concentrations of tumor markers CAE and CA 15-3, was found in patients with pleural effusion.

Al-Jarallah et al. (15) reported that the median serum levels of CA 15-3 or CEA tend to increase depending on the localization of the metastases. The highest serum concentrations of the tumor marker CA 15-3 were found in patients with bone metastases, while the highest serum concentrations of tumor marker CEA were found in patients with metastasis to the lungs, bones or liver. Serum tumor marker CA 15-3 was elevated in more than $90 \%$ of patients with advanced disease and metastasis to the lungs, bones or liver.

Studies by other authors have agreed that the tumor marker CA 15-3 is mainly more sensitive in the presence of bone and visceral metastases. Similar results were obtained in the study by Laessing et al. (13), where they demonstrated elevated serum levels of the tumor markers CA 15-3 and CEA in patients with bone and liver metastases, and lower serum concentrations of tumor markers CA 15-3 and CEA in patients with metastasis to the lungs and lymph nodes.

A correlation between elevated serum levels of tumor markers CEA and CA 15-3 and the disease was shown by Colomer et al. (16). In their study, patients with multiple metastases had a higher proportion of elevated serum levels of tumor marker CA 15-3, compared to patients with metastasis to only one localization (81.2\% compared to $51.4 \%$ ).

The results of this study are similar to the results of other studies performed in the 
world. In this study, taking into account the mammographic and ultrasound features of the changes to the breasts, that are in $72 \%$ of cases classified as BIRADS V, and in 28\% of cases as BIRADS IV, and the values obtained of serum concentrations of tumor markers CA 15-3, it has been shown that tumor marker CA 15-3 has no diagnostic value in the diagnosis of breast cancer, except in exceptional cases of significantly elevated values of tumor marker CA 15-3, when used to find the localization of the primary tumor that has already spread to distant metastasis. We have shown that serum tumor marker CA 15-3 is not elevated in patients with histologically verified breast cancer, and in whom there is no proven presence of regional or distant metastases, but the values of serum concentrations of tumor marker CA 15-3 were significantly elevated in patients with verified breast cancer, axillary and distant metastasis, or associated metastases, and its concentration increases depending on the number of affected organs and organ systems, and on the dissemination of the underlying disease. However, in patients with verified breast cancer and metastases in the axillary lymph nodes, in as many as $64.5 \%$ of cases, the concentrations of the tumor marker CA 15-3 were not increased.

\section{Conclusion}

There is a significant correlation between serum levels of tumor marker CA 15-3 and the presence of metastasis, or the value of tumor marker CA 15-3 and the dissemination of the basic disease. It is certain that a minimally invasive diagnostic test, such as the determination of serum levels of tumor markers, would be very useful in the detection of breast cancer. However, the tumor marker CA 15-3 as such should not be used as it may be elevated in patients with benign changes in the breast, and normal in patients with the presence of primary breast cancer, and even in patients with proven breast cancer and verified metastases in the regional axillary lymph nodes. Mammography and ultrasound are proven effective diagnostic methods in the early detection of cancer (early stage disease) and this significantly affects the prognosis, the treatment, the quality of life and prolonging the life of patients.

Authors' contributions: Conception and design: HF; Acquisition, analysis and interpretation of data: HF, SM, MB, SA; Drafting the article: HF; Revising it critically for important intellectual content: HF, SM.

Conflict of interest: The authors declare that they have no conflict of interest.

\section{References}

1. Malenica B. Immunodiagnosis of breast cancer. In: Fajdić J, editor. Contemporary diagnosis of breast disease, Zagreb: Medical Edition; 2001. p. 211-20.

2. Reynoso G, TM Chu, Holyoke D, Cohen E, Nemoto T, Wang JJ. Carcinoembryonic antigen in patients with different cancers. JAMA. 1972;220(3):361-5.

3. Cooper MJ, Mackie CR, Skinner DB, Moossa AR. A reappraisal of the value of carcinoembryonic antigen in the management of patients with various neoplasms. Br J Surg. 1979;66:120-3.

4. Clarke CA, Whitehead TP, Whitfield AGE. Carcinoembryonic antigen and smoking. J Royal College of Phys London. 1982;16(2):112-3.

5. Wu JT, Nakamura RM, Clinton RS, Beason LK. A comparative study of four serological tumor markers for the detection of breast cancer. In: Wu JT, editor. Human circulating tumor markers: current concepts and clinical applications, Chicago: ASCP Press; 1997. p. 263.

6. Duffy MJ, Shering S, Sherry F, McDermott E, O'Higgins N. CA 15-3: a prognostic marker in breast cancer. Int J Biol Markers. 2000;15(4):330-3.

7. Molina R, Barak V, van Dalen A, Duffy MJ, Einarsson R, Gion M, et al. Tumor markers in breast cancer-European Group on Tumor Markers recommendations. Tumour Biol. 2005;26(6):281-93.

8. Donepudi MS, Kondapalli K, Amos SJ, Venkanteshan P. Breast cancer statistics and markers. J Cancer Res Ther. 2014;10(3):506-11.

9. Basuyau JP, Blanc-Vincent MP, Bidart JM, Daver A, Deneux L, Eche N, et al. Summary report of 
the standards, options and recommendations for the use of serum tumor markers in breast cancer: 2000. Br J Cancer. 2003;89(1):S32-4.

10. Harris L, Fritsche H, Mennel R, Norton L, Ravdin P, Taube S, et al. American Society of Clinical Oncology 2007 Update of recommendations for the use of tumor markers in breast cancer. J Clin Oncol. 2007;25(33):5287-312.

11. Vrdoljak D, Knežević F, Ramljak V. The relation between tumor marker $\mathrm{Ca}$ 15-3 and metastasis in interpectoral lymph nodes in breast cancer patients. Saudi Med J. 2006;27(4):460-2.

12. Berberoglu U, Ceyhan B, Ercakmak N, Sezderdogdu V. The value of new tumor marker CA 15-3 in diagnosis and monitoring of patients with breast cancer. J Islamic Acad Sci. 1989;2:2:113-7.

13. Laessing D, Nagel D, Heinemann V, Untch M, Kahlert S, Bauerfeind I, et al. Importance of
CEA and CA 15-3 during disease progression in metastatic breast cancer patients. Anticancer Res. 2007;27 (4A):1963-8.

14. Berruti A, Tampellini M, Torta M, Buniva T, Gorzegno G, Dogliotti L. Prognostic value in predicting overall survival of two mucinous markers: CA 15-3 and CA 125 in breast cancer patients at first relapse of disease. Eur J Cancer. 1994;30:2082-4.

15. Al-Jarallah MA, Behbehani AE, el-Nass SA, Temim L, Ebraheem AK, Ali MA, et al. Serum CA 15-3 and CEA patterns in postsurgical follow-up, and in monitoring clinical course of metastatic cancer in patients with breast carcinoma. Eur J Surg Oncol. 1993;19:74-9.

16. Colomer R, Ruibal A, Genolla J, Rubio D, Del Campo JM, Bodi R, et al. Circulating CA 15-3 levels in the postsurgical follow-up of breast cancer patients and in non-malignant diseases. Breast Cancer Res Treat. 1989;1:123-33. 